\title{
Erhaltungstherapie mit Histamindihydrochlorid plus IL-2
}

Etwa $40 \%$ bis $50 \%$ der Patienten mit akuter myeloischer Leukämie (AML) im Alter von unter 55 Jahren haben eine gute Prognose. Für Patienten, die älter als 60 Jahre sind, liegt das krankheitsfreie 5-Jahres-Überleben aber nur bei $15 \%$ bis $20 \%$. Für diese Patienten gilt es, einen Kompromiss zu finden zwischen zumutbarer Toxizität der systemischen Therapie und dem Rezidivrisiko. Dabei spielt die Erhaltungstherapie nach Induktion und Konsolidierung eine wichtige Rolle.

In den 1980er Jahren wurde gezeigt, dass das Zytokin Interleukin-2 (IL-2) als Monotherapie komplette Remissionen induzieren kann. In neueren Studien konnte dies aber nicht bestätigt werden. Untersuchungen von Kristoffer Hellstrand und Kollegen führten zu der Erkenntnis, dass oxydativer Stress die IL-2-Immuntherapie bei Krebs behindert und Histamin diesen oxydativen Stress zu reduzieren vermag (Hellstrand K et al., 1994, J Immunol 153: 4940-4947). So führte die Addition von Histamin zu IL-2 in vitro zu einer deutlich besseren T-Zell-Antwort. Auch in klinischen Studien zeigte die Kombination Wirkung: Bereits 2006 wurden die Ergebnisse einer randomisierten Phase-III-Studie veröffentlicht, in der eine Erhaltungstherapie mit IL-2 plus Histamindihydrochlorid (HDC) zu einem signifikant besseren 3Jahres-Überleben führte als keine Erhaltungstherapie (40\% versus 26\%; $p=0,01$; Brune M et al., 2006, Blood 108: 88-96). In dieser Studie waren 320 Patienten (medianes Alter 57 Jahre) nach kompletter Remission randomisiert für zehn 21-Tage-Zyklen täglich mit IL-2 (14.400U/kg KG s.c., bid) plus HDC (0,5mg s.c., bid) oder nicht behandelt worden. Insgesamt wurde die IL-2/HDC-Therapie gut vertragen, erklärte Wolf-K. Hofmann, Mannheim. Hofmann geht davon aus, dass das gute Ansprechen dieser Therapie auch ein Zeichen für die wich- tige Rolle der T-Zell-vermittelten Immunprozesse bei der Kontrolle bzw. Eradikation residueller Leukämiezellen bei der AML ist.

\begin{abstract}
Welche Rolle spielt Histamin in der Immuntherapie bei Krebs Kristoffer Hellstrand, Göteborg/Schweden, erklärte den Mechanismus wie folgt: AML-Blasten generieren über die membranständige NADPH-Oxydase vermehrt reaktive Sauerstoff-Metaboliten (ROM), durch die NK- und T-Zellen gehemmt werden. Unter Einfluss der ROM verlieren die antileukämischen Lymphozyten ihre Zytotoxizität, es werden nicht nur weniger IL-2-Rezeptoren exprimiert, die Zellen gehen teilweise sogar in Apoptose über. IL-2 kann in dieser Umgebung die NK-und T-Zellen auch nicht mehr aktivieren. Wird der $\mathrm{H}_{2}$-Rezeptor an den myeloischen Zellen durch Histamin aktiviert, kommt es zur Hemmung der NADPH-Oxydase und damit sinkt die ROM-Konzentration. So kann die Inhibition der NK- und T-Zellen aufgehoben werden und IL-2 wieder wirken (Hellstrand K et al., 2000, Med Oncol 12: 258-269).

Histamindihydrochlorid (Ceplene ${ }^{\oplus}$ ) ist als Orphan Drug in Kombination mit IL-2 zugelassen zur Erhaltungstherapie bei erwachsenen AML-Patienten während der ersten Remission. $D B$

Quelle: Veranstaltung der MEDA Pharma GmbH \& Co. KG
\end{abstract}

\section{Potenziell kurative Metastasenresektion bei kolorektalem Karzinom}

\section{Lebermetastasen: gutes Ansprechen auf Cetuximab}

Patienten mit metastasiertem kolorektalem Karzinom ( $m C R C$ ) und alleinigem Leberbefall haben durch eine potenziell kurative Metastasenresektion die Chance auf ein Langzeitüberleben. Auch initial inoperable Lebermetastasen können durch eine präoperative Therapie oftmals noch reseziert werden. Wichtig ist jedoch eine stark remissionsinduzierende Behandlung, z.B. unter Einschluss von Cetuximab.

Prospektiv geprüft wurde die präoperative Kombinationstherapie mit Cetuximab (Erbitux ${ }^{\oplus}$ ) in der Phase-II-Studie CELIM, für die 111 Patienten mit primär nicht resektablem $\mathrm{mCRC}$ mit alleiniger Lebermetastasierung rekrutiert wurden. Definiert war die Inoperabilität als Vorhandensein von technisch nicht resektablen oder 5 und mehr Lebermetasta- sen, informierte Ralf Hofheinz, Mannheim. Die Patienten erhielten randomisiert FOLFOX (Folinsäure [FS]/Fluorouracil [FU]/Oxaliplatin) oder FOLFIRI (FS/FU//rinotecan) plus Cetuximab, zunächst für 8 Zyklen. Danach erfolgte die Evaluation auf Resektabilität. Bei technisch weiterhin nicht operablen Metastasen erhielten die Patienten 4 zusätzliche Therapiezyklen. Bei operablen Metastasen wurde die Therapie nach der Resektion für weitere 6 Zyklen fortgesetzt.

Auf die Cetuximab-Kombinationstherapie sprachen $70 \%$ der Patienten mit KRASWildtyp-Tumoren ( $n=67$ ) mit einer partiellen oder kompletten Remission an (Folprecht G et al., 2009, ASCO GI: \#296). Insgesamt konnten 36 von 106 Patienten (34\%) R0-reseziert werden. Im FOLFOX-Arm ( $n=53)$ lag die R0-Resektionsrate bei 38\%, im FOLFIRI-
$\operatorname{Arm}(n=53)$ war sie mit 30\% etwas niedriger. Insgesamt konnte fast die Hälfte der Patienten (46\%) nach präoperativer Therapie einer Intervention - R0- oder R1-Resektion mit oder ohne Radiofrequenzablation - zugeführt und makroskopisch komplett von den Lebermetastasen befreit werden.

Die verblindete Auswertung von Computer- und Kernspintomogrammen der Patienten zu Studienbeginn und nach präoperativer Chemotherapie ergab, dass die Cetuximab-Kombinationstherapie die Resektabilität von Lebermetastasen deutlich verbessert (Bechstein WO et al., 2009, ASCO: \#4091): Waren initial nur 32\% der Patienten als operabel eingestuft worden, stieg dieser Anteil nach Abschluss der Chemotherapie signifikant auf $60 \%$ ( $p<0,0001)$. $K A$

Quelle: Veranstaltung der Merck Serono $\mathrm{GmbH}$ 